\title{
Vascularized bone grafts for post-traumatic defects in the upper extremity
}

\author{
Giovanna Petrella, Daniele Tosi, Filippo Pantaleoni, Roberto Adani \\ Department of Hand Surgery and Microsurgery, University Hospital Policlinico of Modena, Modena, Italy
}

\begin{abstract}
Vascularized bone grafts (VBGs) are widely employed to reconstruct upper extremity bone defects. Conventional bone grafting is generally used to treat defects smaller than 5-6 cm, when tissue vascularization is adequate and there is no infection risk. Vascularized fibular grafts (VFGs) are mainly used in the humerus, radius or ulna in cases of persistent non-union where traditional bone grafting has failed or for bone defects larger than $6 \mathrm{~cm}$. Furthermore, VFGs are considered to be the standard treatment for large bone defects located in the radius, ulna and humerus and enable the reconstruction of soft-tissue loss, as VFGs can be harvested as osteocutaneous flaps. VBGs enable one-stage surgical reconstruction and are highly infection-resistant because of their autonomous vascularization. A vascularized medial femoral condyle (VFMC) free flap can be used to treat small defects and non-unions in the upper extremity. Relative contraindications to these procedures are diabetes, immunosuppression, chronic infections, alcohol, tobacco, drug abuse and obesity. The aim of our study was to illustrate the use of VFGs to treat large post-traumatic bone defects and osteomyelitis located in the upper extremity. Moreover, the use of VFMC autografts is presented.
\end{abstract}

Keywords Bone / Fibula / Graft / Cortico-periosteal / Microsurgery
Correspondence: Giovanna Petrella Department of Hand Surgery and Microsurgery, University Hospital Policlinico of Modena, Largo del Pozzo, 71. Modena 41125, Italy Tel: $+39-59-422-2145$ Fax: +39-59-422-3081 E-mail: giovannapetrella@libero.it

This article was presented at the 36th Scientific Meeting of the Korean Society for Microsurgery on October 28, 2017, in Seoul, Korea.

The authors thank Marisa Mancini, medical illustrator and photographer, for her valuable support in the illustrations and digital content. All the photos and drawings are her own creation.

Received: May 19, 2020 • Revised: September 15, 2020 • Accepted: September 29, 2020

pISSN: 2234-6163 • elSSN: 2234-6171 • https://doi.org/10.5999/aps.2020.00969 • Arch Plast Surg 2021;48:84-90

\section{INTRODUCTION}

Bone grafting is required for numerous bone defects, including those in the upper extremity with non-union or delayed union of a fracture after high-energy trauma associated with significant bony tissue loss, as well as in cases of infection. Bone nailing, external fixators, and compression plates associated with non-vascularized bone grafts (VBGs) are standard tools for the treatment of humerus and radius-ulna diaphyseal bony non-union and lead to good results in most cases. Non-union is frequently caused by poor techniques of osteosynthesis with unsatisfactory skeletal fixation. In addition, bone nailing is contraindicated when there is evidence of an existing or recurring infection, as occurs with conventional bone grafts. Failure is frequently observed in bony defects greater than $6 \mathrm{~cm}$.

Non-VBGs have multiple distinct characteristics that stimulate bone healing. The effectiveness of materials used for grafts is determined based on their osteogenicity, osteoinductivity, and osteoconductivity [1]. It is also mandatory to take into account the mechanical strength and vascular supply to the bone graft material (Table 1). Autologous bone grafts show excellent histocompatibility, osteoconductivity, osteoinductivity and osteogenicity; therefore, they enable bone incorporation into the adjacent host site through the process of "creeping substitution," for 
Table 1. Schematic presentation of properties of different types of bone grafts

\begin{tabular}{lccccc}
\hline Type of graft & Osteoconduction & Osteoinduction & Osteogenesis & Mechanical strength & Vascularity \\
\hline Bone marrow & $+/-$ & + & ++ & - & - \\
Cancellous autograft & $+/+$ & + & ++ & + & + \\
Cortical autograft & + & $+/-$ & + & ++ & - \\
Vascularized & +++ & + & ++ & ++ \\
\hline
\end{tabular}

which reason autologous bone grafting is considered to be the standard of care. The bone graft material is progressively revascularized and finally reabsorbed, allowing new living bone to form; this new bone is then incorporated and remodeled in the host skeleton [2].

VBGs use cortical or cortico-cancellous autografts, harvested with their vascular supply, and are therefore immediately viable. The vascularized free graft (VFG) was first described 45 years ago [3] and this technique is now commonly used in clinical practice. In recent years, significant series of VFGs for forearm $[4,5]$ and humerus reconstruction $[6,7]$ have been reported, in which VFGs are harvested as osteocutaneous flaps [8]. The vascularized medial femoral condyle (VMFC) flap was introduced by Sakai et al. in 1991 [9], who used medial femoral condyle periosteal flaps to treat upper limb non-union fractures [10]. VBGs are incorporated via primary bone healing into the bone recipient site without the need for creeping substitution. This process enables preservation of the structural integrity and mechanical strength of the graft, yielding immediate stability and greater strength. VBG is more efficient than conventional cortico-cancellous grafts due to several advantages. Specifically, the living bone graft enables straightforward and rapid fracture healing by serving as a source of osteogenic cells, promoting vascularization, eliminating infection and enhancing intrinsic stability at the non-union site. VBGs can be harvested from the fibula, iliac crest, distal radius, ribs, scapula, medial femoral condyle, the phalanx of the toe and metacarpal bones.

\section{PATIENTS}

Eighty-five patients underwent surgical reconstruction of posttraumatic bone defects of the upper extremity using VMGs between 1989 and 2018. Patients' mean age was 39 years (range, 16-65 years). VFGs were employed in 74 cases $(87 \%)$ and free VMFC flaps in 11 cases (13\%). All patients had previously undergone multiple interventions for union or to treat an infected site, with an average of four interventions per patient (range, 2-6), with the exception of two cases treated with scaphoid reconstruction.

Osteomyelitis most commonly developed in the humerus and forearm. The average time between the initial trauma and reconstructive surgery was 39 months (range, 4-130 months). The humerus was reconstructed in 31 cases, the radius in 37 cases (in eight cases using a VMFC flap as a corticoperiosteal flap), the ulna in 14 , the scaphoid in two (vascularized medial femoral trochlea osteocartilaginous flaps were used to reconstruct proximal pole scaphoid non-unions) and the metacarpal in one (employing a VMFC flap as an osteoperiosteal flap). The length of the bone defect, considering only cases where a VFG was used, ranged from 6 to $16 \mathrm{~cm}$ (average, $9.4 \mathrm{~cm}$ ). The average length of defects in the humerus was $10.5 \mathrm{~cm}$ and that of defects in the forearm was $8.4 \mathrm{~cm}$.

In nine cases, a VFG was harvested as an osteocutaneous flap. Fibular fixation in the humerus was achieved using a single plate (a reconstruction plate, locking compression plate, or dynamic compression plate in most of the cases treated), although two separate plates and screws were employed in a few cases in the early phase of our experience. For fibular fixation of the radius and ulna, two separate plates were employed, although we currently prefer to use a long plate. Pin staples and screws associated with external fixation systems, such as that of Ilizarov, were employed only in the early cases.

The VMFC grafts were wrapped around the radius in the area of atrophic non-union, and the defect site was also packed with cancellous medial femoral bone grafts. Vascularized corticoperiosteal bone grafts were closed with stitches or suture anchors. The previous plates were left in situ in seven cases, in which only the screws located in the area of non-union were removed.

\section{DISCUSSION}

Extensive bone loss involving the humerus, radius and ulna can be managed with various surgical techniques.

The indications for VBGs fall into two major categories (Table 2 ). The first indication is for bone gaps greater than $6-8 \mathrm{~cm}$, which most often occurs in patients with post-infectious or post-traumatic bone damage and after oncological resection. The second indication is for bony defects in which a biological failure of bony healing has taken place, such as recalcitrant nonunion of fractures, congenital pseudoarthroses or osteonecrosis. 
Table 2. Indications for free vascularized fibula grafting

\begin{tabular}{ll}
\hline Segmental bone defects & Traumatic bone loss \\
greater than 6-8 cm & Tumor resection \\
& Osteonecrosis \\
& Osteomyelitis \\
Biological failure of bony healing & Persistent non-union \\
& Infected non-union \\
& Congenital pseudoarthrosis \\
\hline
\end{tabular}

Fig. 1. Necrotic radius, infected bone debridement

Case 1. The radius had been operated on four times within 3 years, with complete resorption of the conventional bone graft (A). After removing the plate $(B)$, extensive debridement and excision of all necrotic bone were performed (C), creating a segmental defect requiring reconstruction (D).
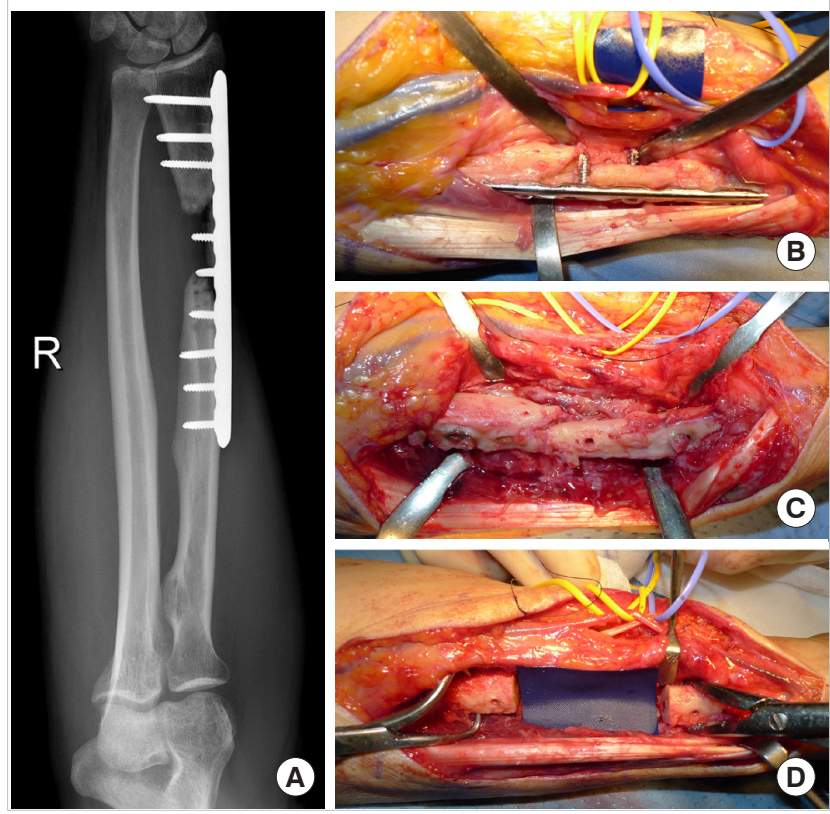

In order to reduce the complication rates, accurate patient intake with a thorough clinical assessment is fundamental; additionally, local wound conditions, the etiology of trauma and previous surgical outcomes should be carefully evaluated. VBGs could be indicated for interventions in the ankle, tibia, femur, ulna, radius, humerus, clavicle and spine.

Various techniques and donor sites may be chosen to reconstruct bone losses of the upper extremity. However, the specific features of VFGs make them suitable for excellent bone reconstruction in this anatomical region, as it is necessary to ensure fit of the diameter of the recipient bone with that of the donor site. The shape and diameter of the fibula are similar to those of the forearm bones, while the humerus requires further augmentation with a bridging plate. Once the fibula is positioned at the recipient site, it is capable of undergoing a remodeling process to sustain the new functional load, which appears as hypertro-

\section{Fig. 2. Free fibula flap with periosteal sparing}

Case 1. (A) A sleeve of vascularized periosteum is retained with a vascularized fibular graft. (B) Plate was fixed using a single locking compression plate. The sleeve of vascularized periosteum was cuffed around the recipient bone (white arrows) to enhance healing at the junction site.
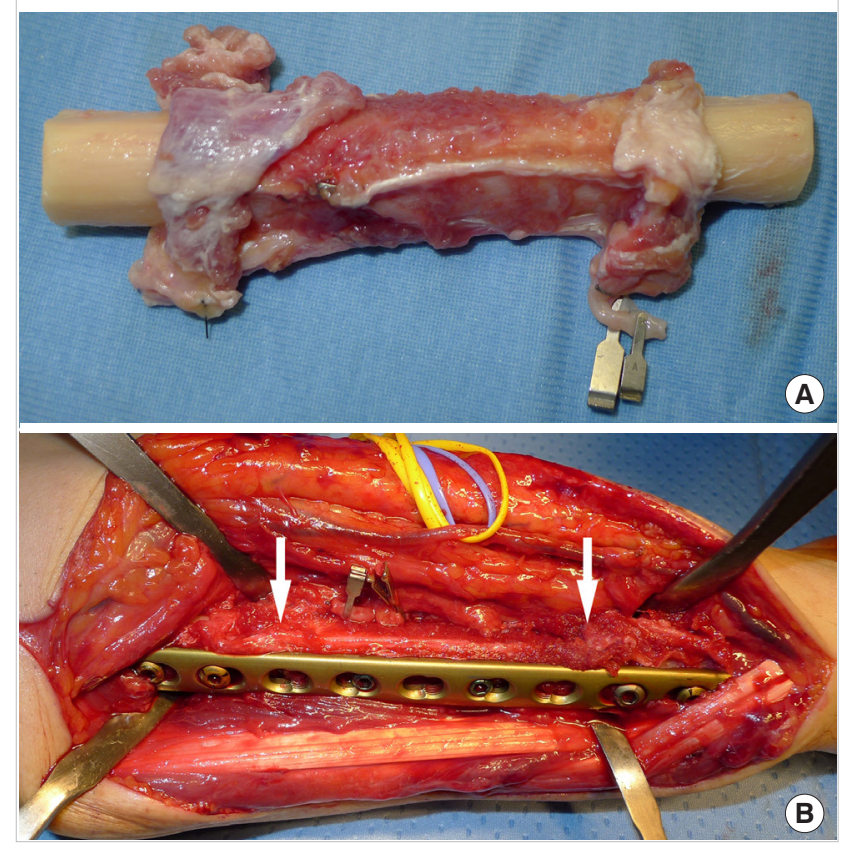

phy of the humerus on radiography.

The mean healing time reported in the literature is 6 months for the humerus $[6,7]$ and 4.8 months for the radius or ulna $[4,5]$. To reduce the time to union, the authors performed the following procedures: (1) carrying out meticulous debridement of the necrotic/infected bone, as bone involvement may be more extensive than anticipated based on preoperative imaging studies (Fig. 1); (2) saving a periosteal flap, which should overlap the junction between the fibula and host bone, forming an additional source of new bone production (Figs. 2, 3); (3) using a preplate for radius or ulna graft reconstruction, as it makes the implant more handy; (4) using a single bridging plate to reduce the risk of stress fractures due to weak points between plates, particularly in the case of humerus reconstruction (Fig. 4); (5) employing uni-cortical screws to fix the VFG to the plate; and (6) realigning the distal radio-ulnar joint in forearm reconstruction.

A fixation method should be chosen that optimizes the local blood supply and supplements and protects the implanted grafts [11]. Plates in the upper extremity are the method of choice to achieve fixation of the grafted fibula. We propose three different techniques to achieve good contact at the graft and host junction in the upper extremity: (1) step-cut osteotomy to maximize bone contact, which is technically demanding and mainly indicated for the forearm (Fig. 5); (2) transverse osteotomy, 


\section{Fig. 3. Outcomes of case 1}

(A) Radiographic evaluation after 6 months. $(B, C)$ Clinical evaluation: pronosupination is maintained (right hand).
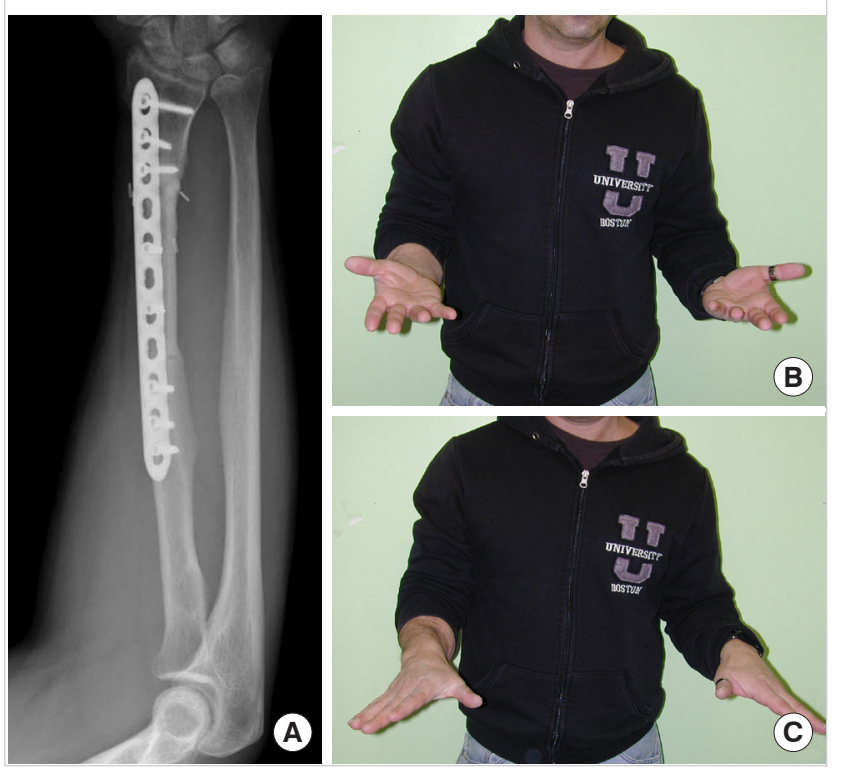

Fig. 4. Humerus non-union

(A) Recalcitrant non-union of the humerus, with multiple failed operations. (B) Reconstruction was performed with a vascularized fibular graft (VFG). Internal fixation was achieved with a locking compression plate. (C) Twelve months after surgery, $\mathrm{X}$-rays revealed union and relevant hypertrophy of the VFG.
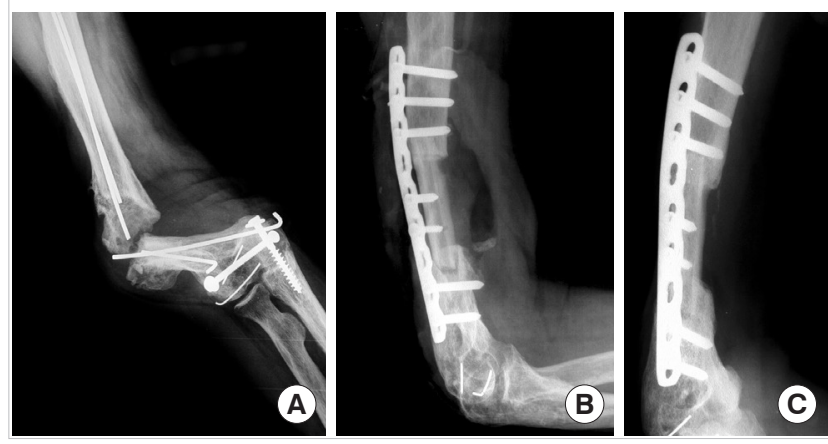

which is technically straightforward and indicated for forearm reconstruction to manage forearm rotation, if there is a smaller surface of bone contact compared to step-cut osteotomy (Fig. 6); and (3) intramedullary press-fit, which is technically easy and indicated mainly for proximal humerus reconstruction where it guarantees optimal bone contact with good stability (Fig. 7). The optimal timing for bone reconstruction in the presence of infection is still controversial [12]. In case of active septic non-union we prefer a staged repair, with the application of antibiotic cement with or without vacuum-assisted closure therapy, instead of one-stage reconstruction after radical debridement of the infection (Fig. 8).

\section{Fig. 5. Step-cut osteotomy}

(A) Schematic representation of step-cut osteotomy for ulna reconstruction. (B) X-ray examination showing postoperative outcomes.
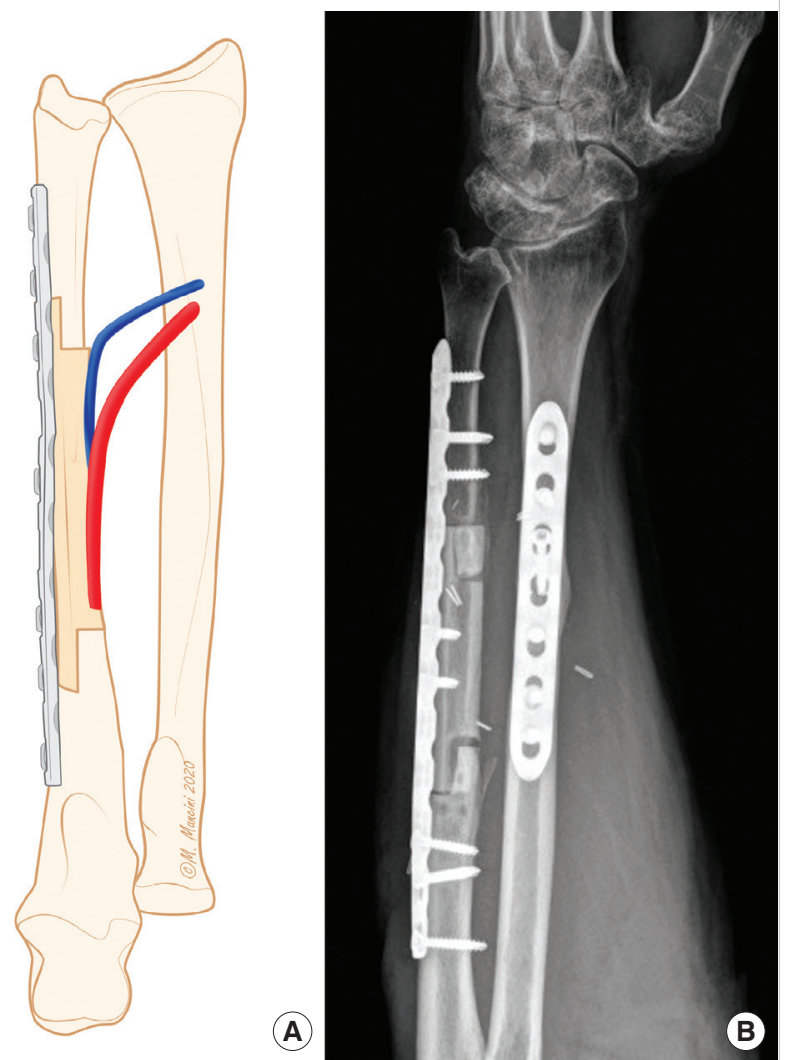

Fig. 6. Transverse osteotomy

(A) Schematic representation of transverse osteotomy for radius reconstruction. (B) Preplating the vascularized fibular graft. (C) Intraoperative view after osteosynthesis with a locking compression plate. (D) X-ray examination showing postoperative outcomes.

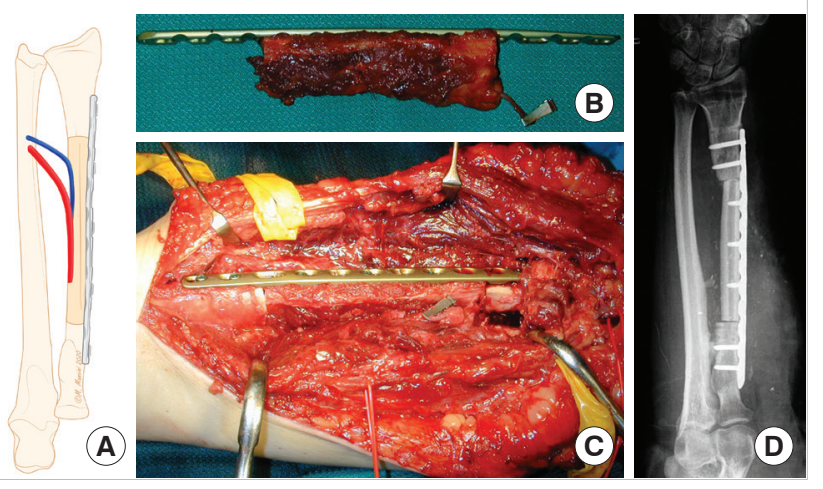

Careful patient selection is essential in order to reduce the complication rate. Stress fractures of the graft are a frequently occurring complication, which may be associated with hardware breakage in $15 \%$ to $20 \%$ of cases. Generally, complications occur within the first year postoperatively $[4,6,13]$. Chronic infections; diabetes; immunosuppression; alcohol, tobacco, and drug 
abuse; and obesity are relative contraindications for vascularized graft procedures. Local wound conditions, the cause of the trauma, and previous surgical outcomes should also be carefully considered.

VFMC flaps represent an alternative approach for treating atrophic non-union (Fig. 9). The periosteum is well known to be a source of osteochondral progenitor cells, which contribute to new bone formation; this fact provides a rationale for using periosteal grafts instead of or along with bone grafting [14]. It is possible to elevate periosteal flaps with no inclusion of the underlying bone, or with cortical and cortico-cancellous bone. Corticoperiosteal flaps harvested from the medial condylar region of the femur are a source of thin, pliable, highly osteogenic vascularized tissue with minimal donor site morbidity. Due to its degree their flexibility, these flaps may be wrapped around the radius or ulna to form a "biological sleeve" (Fig. 10) [15]. Subperiosteal removal of two or three longitudinal 2-mm-wide

\section{Fig. 7. Intramedullary press-fit insetting}

(A) Schematic representation of an intramedullary press-fit for humerus reconstruction. (B) Intraoperative result after osteosynthesis with a long bridging plate. (C) X-ray examination showing postoperative outcomes.
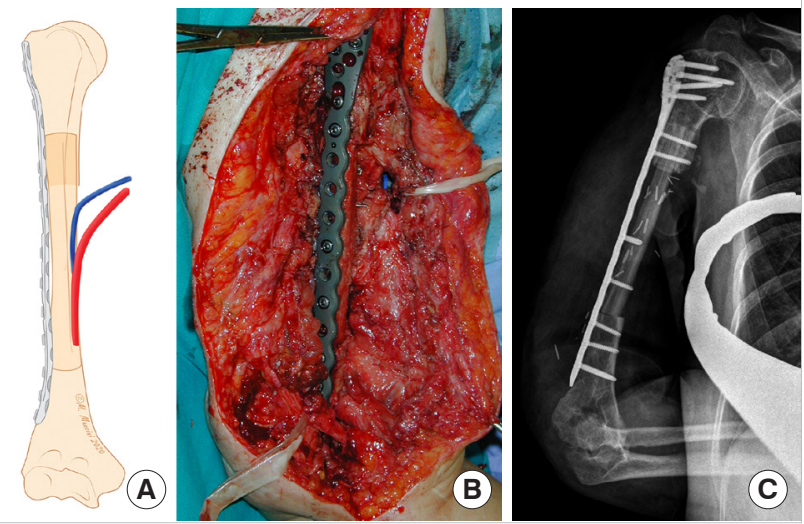

strips of cortex is useful for flap insetting [16]. The mean time of bone graft healing reported in our case series (3.9 months) is comparable to the intervals that have been reported in the literature (Fig. 11) [14,15]. Cortico-periosteal flaps are indicated for recalcitrant, long-standing non-union and other cases with poorly vascularized soft tissue beds, in which the classic fibular flap is not ideal based on the size of the defect. The success of

\section{Fig. 9. Recalcitrant radius non-union}

Case 3. Radiographic appearance.

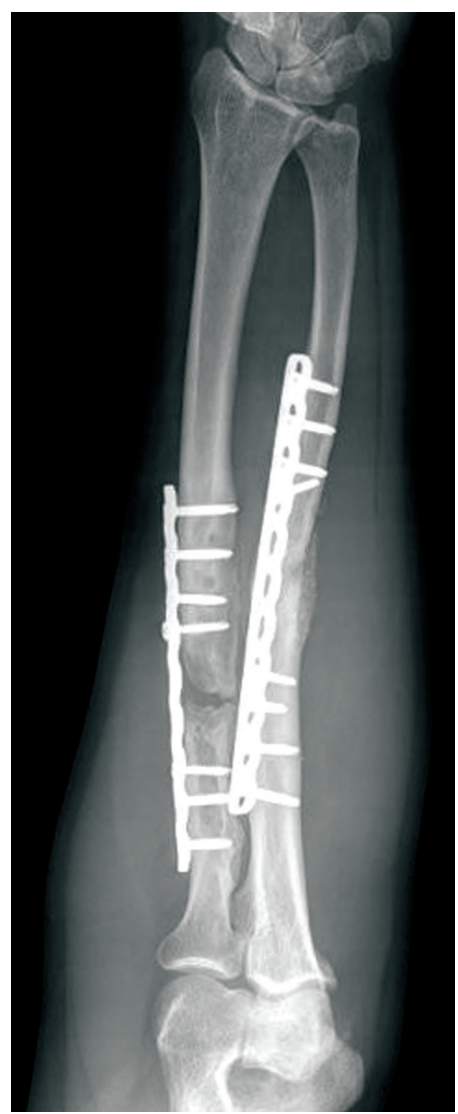

\section{Fig. 8. Staged repair in forearm infection}

Case 2. $(A, B)$ Open ulnar fracture treated 4 months prior with a plate, with clinical and radiological features of severe infection in the forearm. (C, D) After debridement, a cement spacer was applied. (E) Three months after, vascularized free graft reconstruction was planned. (F) Radiological result of the reconstructed ulna, 12 months after surgery.
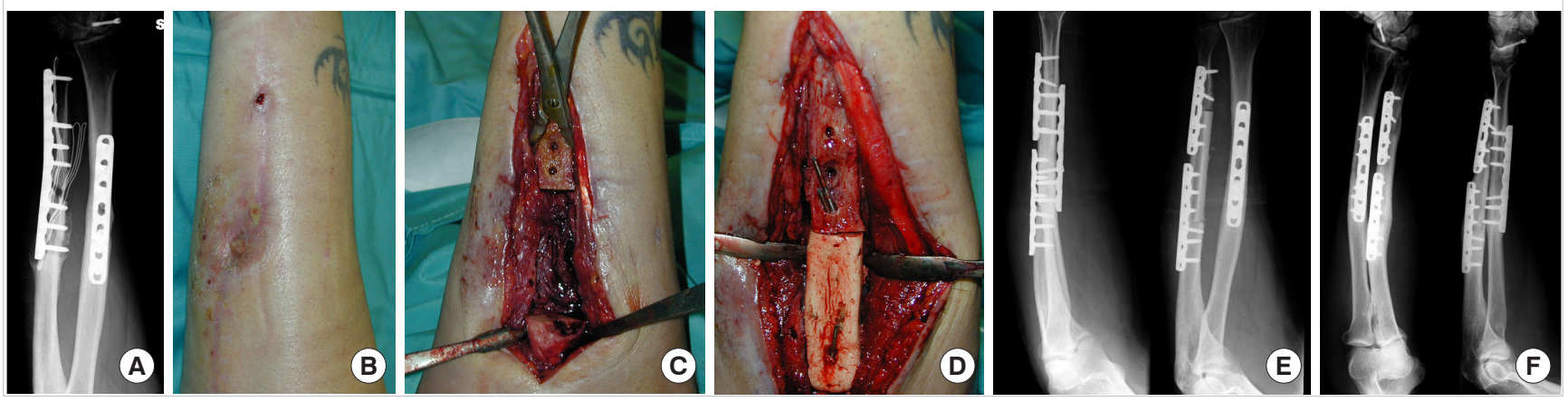


\section{Fig. 10. Corticoperiosteal flap}

Case 3. (A) A thin corticoperiosteal flap is harvested from the medial surface of the supracondylar region. The graft is cut with the aid of a chisel (proceeding from distal to proximal to avoid vessel injury). (B) Good vascular supply of the flap after releasing the tourniquet. (C) The medial femoral condylar periosteal flap is smaller and more flexible and may be easily tailored to irregular defects. (D) Subperiosteal resection of two or three 2-mm-wide strips of cortical layer allows easier flap wrapping around the bone graft. (E) The flap is placed around the radius and fixed with wire or nonabsorbable suture, and microvascular anastomosis is subsequently performed to the adjacent vessels.
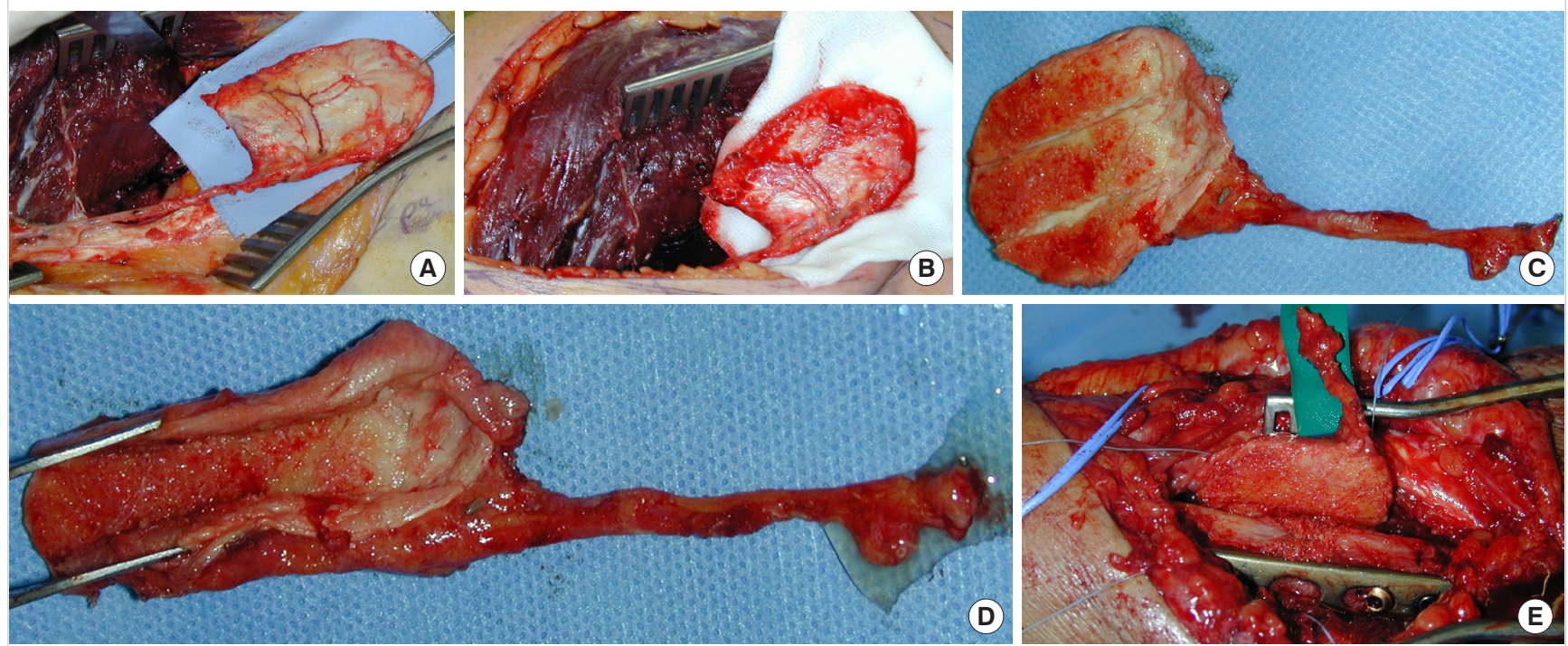

\section{Fig. 11. Outcomes of case 3}

Radiographic (A) and clinical $(B, C)$ outcomes at 4 months of follow-up.
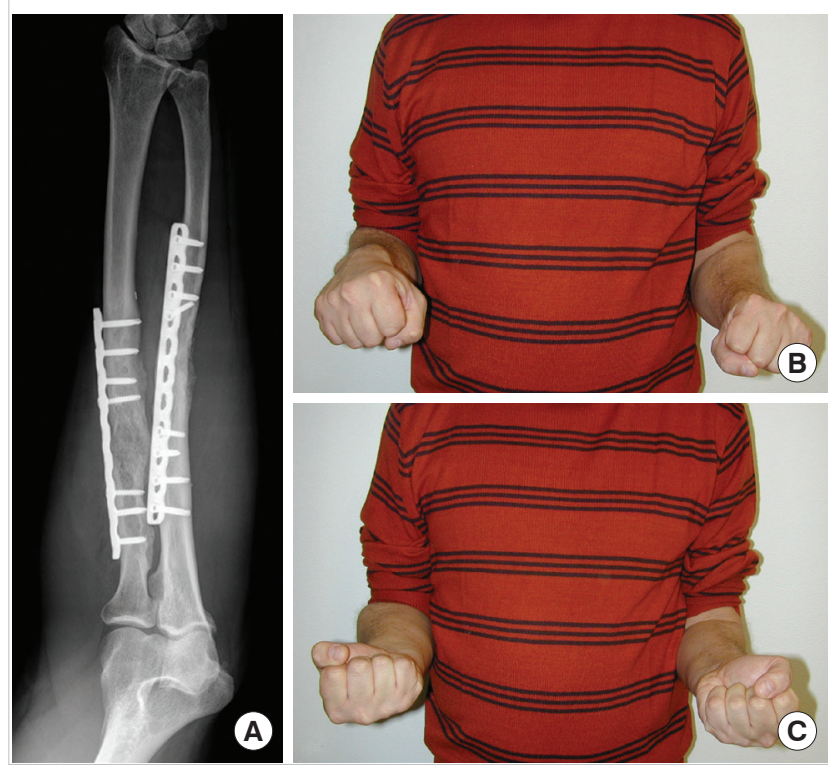

these flaps under such circumstances is due to multiple reasons: first, vascular flap-induced angiogenesis promotes healing and improves the ability to fight an infection, and second, these flaps are osteogenic, which is crucial for new bone formation in nonunion [17]. It is rare for donor-site morbidity and other complications to occur.

Stable-plate internal fixation is the best choice for the upper extremity. VFGs should be considered for the reconstruction of bone defects larger than 6-7 cm and complex defects necessitating bone and soft tissue reconstruction. VFMCs are indicated in cases of recalcitrant, long-standing non-union to maximize the healing rate due to their well-vascularized, highly osteogenic character, and where defect size does not justify the use of a VFG. Unlike other VFG techniques, VFMC flaps are thin and pliable, enabling them to conform better to the recipient site and/or to wrap around the tubular bone non-union sites are located. VFMC corticoperiosteal flaps effectively promote early bony union in cases of persistent, long-standing non-union, where conventional bone graft techniques have failed.

\section{NOTES}

\section{Conflict of interest}

No potential conflict of interest relevant to this article was reported.

\section{Ethical approval}

The study was performed in accordance with the principles of the Declaration of Helsinki.

\section{Patient consent}

The patients provided written informed consent for the publication and the use of their images. 


\section{Author contribution}

Conceptualization: G Petrella, D Tosi, R Adani. Data curation: G Petrella, F Pantaleoni, R Adani. Formal analysis: D Tosi, F Pantaleoni, R Adani. Methodology: all authors. Project administration: R Adani. Visualization: F Pantaleoni, R Adani. Writing - original draft: G Petrella, D Tosi, R Adani. Writing - review \& editing: all authors.

\section{ORCID}

$\begin{array}{ll}\text { Giovanna Petrella } & \text { https://orcid.org/0000-0001-5211-688X } \\ \text { Daniele Tosi } & \text { https://orcid.org/0000-0002-4894-206X } \\ \text { Filippo Pantaleoni } & \text { https://orcid.org/0000-0001-7437-6236 } \\ \text { Roberto Adani } & \text { https://orcid.org/0000-0003-0181-333X }\end{array}$

\section{REFERENCES}

1. Klifto CS, Gandi SD, Sapienza A. Bone graft options in upper-extremity surgery. J Hand Surg Am 2018;43:755-61.

2. Khan SN, Cammisa FP Jr, Sandhu HS, et al. The biology of bone grafting. J Am Acad Orthop Surg 2005;13:77-86.

3. Taylor GI, Miller GD, Ham FJ. The free vascularized bone graft: a clinical extension of microvascular techniques. Plast Reconstr Surg 1975;55:533-44.

4. Adani R, Delcroix L, Innocenti M, et al. Reconstruction of large posttraumatic skeletal defects of the forearm by vascularized free fibular graft. Microsurgery 2004;24:423-9.

5. Safoury Y. Free vascularized fibula for the treatment of traumatic bone defects and nonunion of the forearm bones. J Hand Surg Br 2005;30:67-72.

6. Adani R, Delcroix L, Tarallo L, et al. Reconstruction of posttraumatic bone defects of the humerus with vascularized fibular graft. J Shoulder Elbow Surg 2008;17:578-84.

7. Chhabra AB, Golish SR, Pannunzio ME, et al. Treatment of chronic nonunions of the humerus with free vascularized fibula transfer: a report of thirteen cases. J Reconstr Micro- surg 2009;25:117-24.

8. Noaman HH. Management of upper limb bone defects using free vascularized osteoseptocutaneous fibular bone graft. Ann Plast Surg 2013;71:503-9.

9. Sakai K, Doi K, Kawai S. Free vascularized thin corticoperiosteal graft. Plast Reconstr Surg 1991;87:290-8.

10. Burger HK, Windhofer C, Gaggl AJ, et al. Vascularized medial femoral trochlea osteocartilaginous flap reconstruction of proximal pole scaphoid nonunions. J Hand Surg Am 2013;38:690-700.

11. Toros T, Ozaksar K. Reconstruction of traumatic tubular bone defects using vascularized fibular graft. Injury 2019 Aug 14 [Epub]. https://doi.org/10.1016/j.injury.2019.08.013.

12. Arai K, Toh S, Tsubo K, et al. Complications of vascularized fibula graft for reconstruction of long bones. Plast Reconstr Surg 2002; 109:2301-6.

13. Malizos KN, Fyllos A, Varytimidis S, et al. Tips to secure healing at the free vascularised fibular graft-to-host bone junction. Injury 2019;50 Suppl 5:S46-9.

14. Bakri K, Shin AY, Moran SL. The vascularized medial femoral corticoperiosteal flap for reconstruction of bony defects within the upper and lower extremities. Semin Plast Surg 2008;22:228-33.

15. Kakar S, Duymaz A, Steinmann S, et al. Vascularized medial femoral condyle corticoperiosteal flaps for the treatment of recalcitrant humeral nonunions. Microsurgery 2011;31:8592.

16. Rodriguez-Vegas JM, Delgado-Serrano PJ. Corticoperiosteal flap in the treatment of nonunions and small bone gaps: technical details and expanding possibilities. J Plast Reconstr Aesthet Surg 2011;64:515-27.

17. Choudry UH, Bakri K, Moran SL, et al. The vascularized medial femoral condyle periosteal bone flap for the treatment of recalcitrant bony nonunions. Ann Plast Surg 2008; 60:174-80. 